\title{
ELECTRIC-MAGNETIC STRUGGLE IN QGP, DECONFINEMENT AND BARYONS
}

\author{
Edward Shuryak*t \\ Department of Physics and Astronomy, University at Stony Brook, \\ Stony Brook NY 11794 USA \\ E-mail: shuryak@tonic.physics.sunysb.edu
}

\begin{abstract}
We argue that quite unusual properties of Quark-Gluon Plasma in the RHIC temperature domain $T=(1-2) T_{c}$ are consequences of the approximate equilibrium between electric and magnetic sectors reached above the deconfinement temperature. Already classical study of few body motion in a electric-magnetic plasma shows abnormally large scattering rate due to the so called "magnetic bottle" effect. Molecular dynamics simulation have found that equal mixture of electric and magnetic quasiparticles do produce plasmas of small viscosity and even smaller diffusion rate, comparable to what is needed to explain RHIC data and also to what follows from AdS/CFT. As a separate issue, we point out that right above $T_{c}$ there should be surviving s-wave baryons made of quarks $(N, \Delta)$, as well as adjoint objects, 3-gluon and 3-monopole chains (the latter being nothing else but "calorons" or finite-T instantons).
\end{abstract}

Critical Point and Onset of Deconfinement 4th International Workshop

July 9-13 2007

GSI Darmstadt,Germany

\footnotetext{
${ }^{*}$ Speaker.

${ }^{\dagger}$ The talk is based on several works done in collaboration with J-F.Liao
} 


\section{Why is sQGP at RHIC such a perfect fluid?}

A realization $[1,2,3]$ that QGP at RHIC is not a weakly coupled gas but rather a strongly coupled liquid has lead to a paradigm shift in the fi eld. It was extensively debated at the "discovery" BNL workshop in 2004 [4] (at which the abbreviation sQGP was established) and multiple other meetings since.

There is no need to repeat any of the arguments, except to note key new observations. Experimental fi ndings indicating that charm quarks are as strongly quenched as light (gluonic) jets, and participate in elliptic flow, have shown that not only viscosity $\eta$ but heavy quark diffusion constant $D$ is anomalously small [5]. Further conformations of conical flow from quenched jets, suggested in $[6,7]$ came both from RHIC (3-particle correlations from PHENIX and STAR) and from impressive AdS/CFT calculations $[8,9]$. All these facts show that jet quenching mechanism is very different from the perturbative picture of the gluon emission: it is rather an emission of intense sound/wake waves as pointed out in [7].

In the intervening years we had to learn a lot, some new some from other branches of physics which happened to have some experience with strongly coupled systems. Those range from quantum gases to classical plasmas to string theory. In short, there seem to be not one but actually two diffi cult issues we are facing:

(i) to understand whether QGP is (or is not) strongly coupled in the quasi-conformal regime, at $T>2 T_{c}$; (ii) to understand physics near the deconfi nement transition, at $T \approx T_{c}$.

Comparison of the results of the AdS/CFT correspondence - adequate for quasi-conformal strongly coupled plasma - to data (see the end of section 5) is encouraging enough to think that the answer to (i) may be positive. This created a lot of excitement, especially among string theorists.

In this talk we will however focus on the second question (ii), trying to relate the observed "perfect fluidity" with the confi nement/deconfi nement transition via electric-magnetic duality arguments. (The reader who wants to jump to the main qualitative argument may go directly to section 4).

\section{Electric-magnetic struggle}

Let me start by explaining what I mean by "struggle" in the title. A charge entering a region of space with a magnetic fi eld has large chances to make fraction of a Larmor circle and get out. Thus the Lorentz force would transfer momentum in a way producing a pressure on a magnetic field. Therefore "electrically dominated" matter have tendency to expel magnetic fi eld into flux tubes. We know this is the case in superconductors or in (e.g. Solar) plasmas.

According to well known t'Hooft-Mandelstamm scenario, the QCD confi ned phase is a "dual superconductor", in which some magnetically charged objects are Bose-condensed. By duality, now it is the electric fi eld which is subdominant and thus expelled from volume into flux tubes. From tension and also size of the "QCD strings", as we used to call them, we know that the pressure on it is quite substantial.

The picture proposed in [10] has three main ideas:

(i) that transition from magnetically dominated confi ned phase to electrically dominated wQGP at high $T$ must proceed via a region of approximate electric-magnetic equilibrium; 


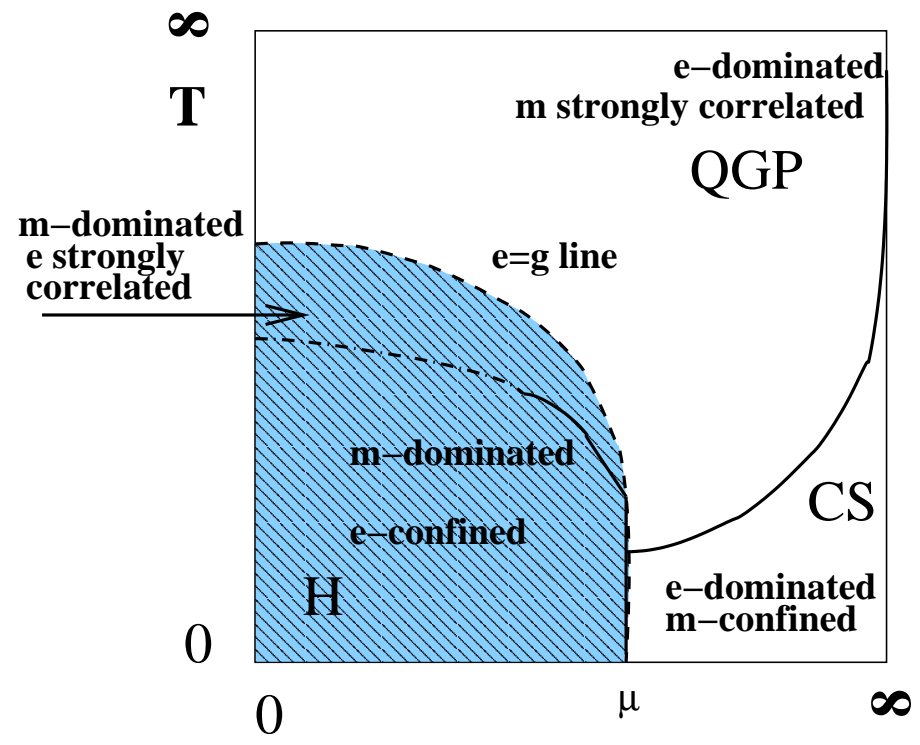

Figure 1: (color online) A schematic phase diagram on a ("compactified") plane of temperature and baryonic chemical potential $T-\mu$. The (blue) shaded region shows "magnetically dominated" region $g<e$, which includes the e-confined hadronic phase as well as "postconfined" part of the QGP domain. Light region includes "electrically dominated" part of QGP and also color superconductivity (CS) region, which has echarged diquark condensates and therefore obviously m-confined. The dashed line called " $\mathrm{e}=\mathrm{g}$ line" is the line of electric-magnetic equilibrium. The solid lines indicate true phase transitions, while the dash-dotted line is a deconfinement cross-over line

(ii) this must happen in the deconfi ned phase which however has signifi cant fraction of magnetic quasiparticles in a "hormal" (uncondenced) phase;

(iii) in this region Dirac condition demands equality of electric and magnetic couplings: this both must be large.

A phase diagram ${ }^{1}$ explaining this pictorially is shown in Fig.1: its blue-shaded part is "magnetically dominated" region at lower $T, \mu$, while white is "electrically dominated" region at large $T$ and/or ${ }^{2} \mu$. They are separated by 'E-M equilibrium" line, which we defi ne by the condition that couplings of both interactions are equal ${ }^{3}$

$$
g_{e}^{2} / 4 \pi \hbar c=g_{m}^{2} / 4 \pi \hbar c=1
$$

The last equality follows from the celebrated Dirac quantization condition

$$
\frac{g_{e} g_{m}}{4 \pi \hbar c}=\frac{n}{2}
$$

\footnotetext{
${ }^{1}$ The arguments use continuity of the transition and so they are most adequate either for pure gauge theory at $N_{c}=2$ or QCD with quarks in which transition is a crossover. Large $N_{c}$ case involves large jump in densities of all components which obscure the picture.

${ }^{2}$ Color superconductor at large density is electrically dominated by diquarks while magnetic objects are confi ned there.

${ }^{3}$ We use fi eld theory notations, in which $g_{e}, g_{m}$ are electric and magnetic couplings, e/m duality transformation is $\tau->-1 / \tau$ where $\tau=\theta / 2 \pi+i 4 \pi / g_{e}^{2} \cdot g_{e}=g$ and $\hbar=c=1$ elsewhere.
} 
with $n$ being an integer". The "magnetic-dominated" low- $T$ (and low- $\mu$ ) region (i) can in turn be subdivided into the confining part (i-a) in which electric fi eld is confi ned into quantized flux tubes by magnetic condensate [11], and a new "postconfinement" region (i-b) at $T_{c}<T<T_{E=M}$ in which the electric quasiparticles - quarks and gluons - already exist but they are heavy and still sub-dominant in number as compared to magnetic ones. This picture, among many other new viewpoints, opens a completely new perspectives on the role of electric flux tubes: they do not disappear at $T=T_{c}$ but are rather at their maximum.

Besides equal couplings, the equilibrium region is also presumably characterized by comparable densities as well as masses of both electric and magnetic quasiparticles. In QCD the issue is complicated by the fact that E-M duality is far from perfect, with different spins of electric (gluons and quarks) and magnetic quasiparticles. However other theories - especially $\mathscr{N}=4$ supersymmetric YM - have perfect self-duality of electric and magnetic description: it is also conformal and has no confi nement to complicate the picture, while $\mathrm{E}$ and $\mathrm{M}$-dominated parameter regions do exist 5 .

If so, there should be be uncondensed magnetic objects above $T_{c}$ as well. And indeed, lattice studies show that electrically charged particles - quarks and gluons - are getting heavier as we decrease $T$ toward $T_{c}$, while monopoles gets lighter and more numerous.

The magnetic screening mass, although absent perturbatively, is nonzero, and even exceeds the electric one close to $T_{c}$ (as shown e.g. by Nakamura et al [12]). ${ }^{6}$ These screening masses as well as estimates of the densities of electric and magnetic objects, leads to the location of $E-M$ equilibrium [10] at

$$
T_{E=M} \approx(1.2-1.5) T_{c}=250-300 \mathrm{MeV}
$$

Seiberg-Witten solution for the N=2 SYM is an excellent example of how transition from one language to another is supposed to work, as the electric and magnetic couplings run in the opposite directions. And indeed, as one approaches the deconfi nement transition the electrically charged particles - quarks and gluons - are getting heavier and heavier while the magnetic objects - monopoles and/or dyons - gets lighter and more numerous.

(The stumbling difference between supersymmetric models and QCD is of course a protection of any Higgsed vacuum by supersymmetry in the former cases, making them all degenerate and thus stable. We cannot discuss in detail a QCD setting: the reader may simply imagine a generic fi nite- $T$ confi guration with a nonzero mean $\left\langle A_{0}\right\rangle$, an adjoint Higgsing leaving $N_{c}-1 \mathrm{U}(1)$ massless gauge fi elds. These U(1)'s corresponds to magnetic charges of the monopoles. In AdS/CFT language one may simply considered $N_{c}$ branes to be placed not at exactly the same point in the orthogonal space.)

\section{Static potentials and flux tubes}

At $T=0$ static potential between heavy quarks is the sum of the Coulomb and the confining

\footnotetext{
${ }^{4}$ Which we put to $\mathrm{n}=2$ because of a presumed adjoint color representation of relevant monopoles.

${ }^{5}$ Not in the phase diagram, as in this theory couplings are independent of $T, \mu$.

${ }^{6}$ Note that electric mass is $M_{E} \sim g T$ is larger than the magnetic one $M_{M} \sim g^{2} T$ at high $T . M_{E}$ however vanishes at $T_{c}$ while $M_{M}$ smoothly grows into confi ned phase toward $T=0$.
} 
$\sim \sigma(T=0) r$ potentials. At nonzero $T$ expectations of the Wilson or Polyakov lines with a static quark pair defi ne the free energy potential $F(T, r)$, which at the deconfi nement point $T=T$ has vanishing string tension $\sigma\left(T_{c}\right)=0$.

However the energy or entropy separately ${ }^{7}$ continue to have linear part in the "postconfi nement" domain $T_{C}<T<T_{E=M}$, as follows from lattice data [13]. Quite shockingly, the tension term in $\mathrm{E}(\mathrm{T}=\mathrm{Tc}, \mathrm{r})$ is not smaller but actually twice larger than the zero temperature tension $\sigma(T=0)$ ! The total energy added to a pair till it fi nally breaks into two objects is surprisingly large: $E\left(T=T_{c}, r \rightarrow \infty\right) \sim 4 \mathrm{GeV}$, while the corresponding entropy as large as $S\left(T=T_{c}, r \rightarrow \infty\right) \sim 20$.

Where all this huge energy and entropy comes from, at $T_{c}$ and in the "postconfi nement" domain? Linear dependence on $r$ hints that it must still be a string-like object connecting two static charges. Can it be that magnetically dominant plasma admits (metastable) electric flux tubes solutions? The answer to this question was given in our recent paper [14]. Indeed, whether monopoles are condensed or not is not so crucial: what is important is the relation between their energy and the repulsive potential of the flux tube. At high enough $T$ flux tube solution disappears, and the critical condition we found roughly does correspond to the upper limit of the "postconfi nement" domain.

\section{The "magnetic bottle" effect}

Before we present manybody studies of plasmas made of electrically and magnetically charged quasiparticles, we would like to explain the essence of the effect using the smallest number of particles possible. The minimal arrangement shown in Fig2(a) includes two static particles of one kind (say +1 and -1 electric charges) and one dynamical particle of the other kind (a monopole of any sign).

Naively one may think that with a velocity $\vec{v}$ (the arrow) looking away from both charges there are no chances of a collisions with them, with any appreciable momentum exchange. However due to the Lorentz force, monopoles with not too large velocity rotate around the electric fi eld line (dashed) while drifting along it. As the monopole approaches one of the charges, the fi eld gets stronger and the Larmor radius smaller: the motion gets confi ned to the so called Poincare cone and collision happens. Effective repulsion (see quantum discussion below) pushes the particle away from the charge, only to repeat the same thing at the other charge.

By duality, the same will happen for a charges surrounded by two monopoles. The principle is thus the same as in famous Budker's "magnetic bottle" invented precisely for ion trapping: only in this case the dynamical particle has electric charge while the static charges are substituted by small coils. The reason a particle is effectively repelled from high fi eld regions inside the coil is due to conservation of adiabatic invariants, and is not absolute.

In summary, in signifi cant part of the phase space a monopole is classically trapped, and cannot avoid periodical head-on collisions with the charges. As a result, particle diffusion is strongly reduced, and a plasma made of electric and magnetic charges can move collectively even for rather small systems.

\footnotetext{
${ }^{7}$ Related as usual by $F=E-T S, S=-\partial F / \partial T$.
} 


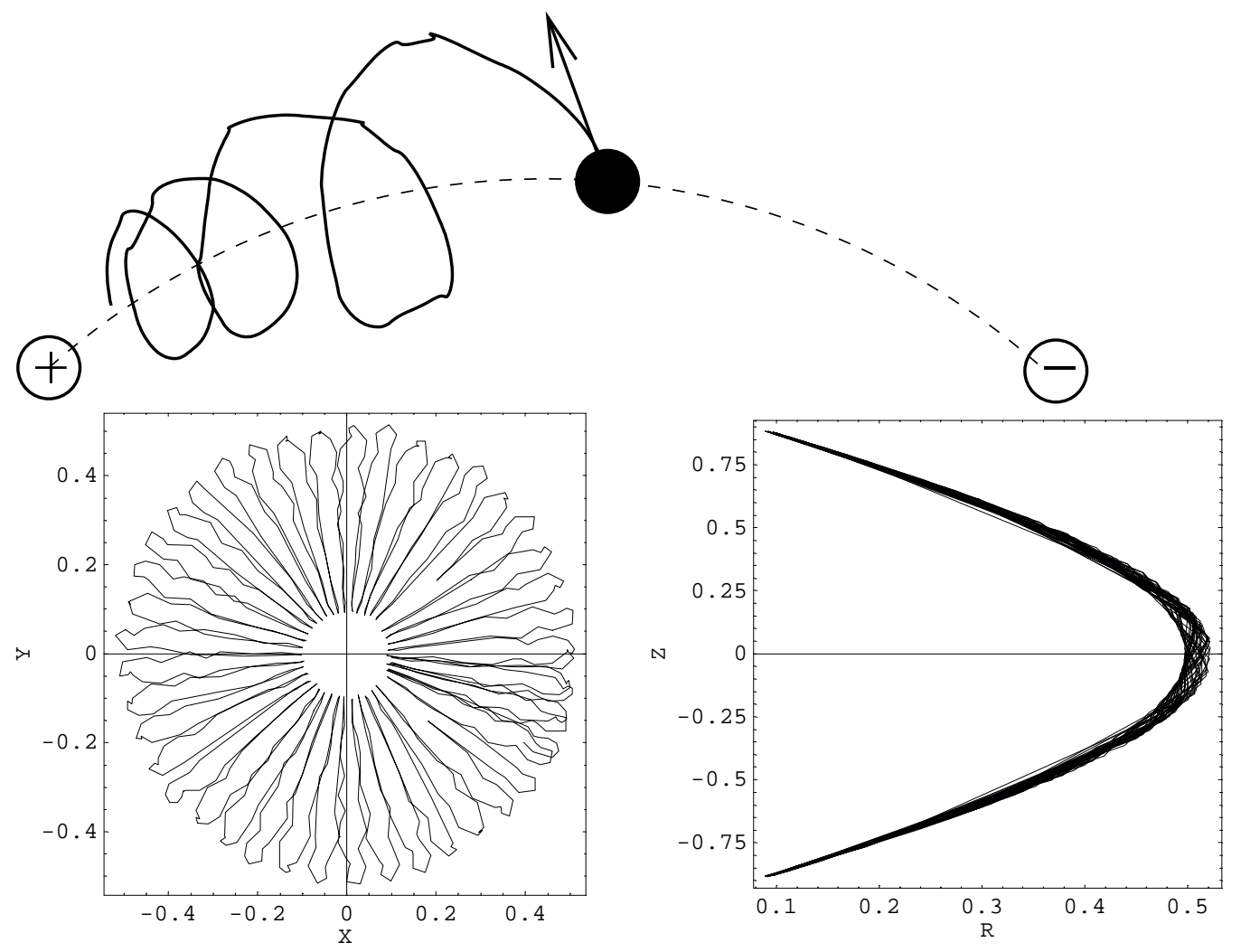

Figure 2: (a) Schematic setting of a monopole moving in a static electric field of two charges \pm 1 . (b) A sample of real trajectory projected on $\mathrm{x}-\mathrm{y}$ plane (left panel) and projected on R-z plane (right panel) (with $R=\sqrt{x^{2}+y^{2}}$ being transverse radius).

Addressing the same problem quantum mechanically, one has the following Hamiltonian for the monopole:

$$
\hat{\mathscr{H}}=\frac{\left(\vec{p}+g \vec{A}_{e}\right)^{2}}{2 m}
$$

Here $\vec{A}_{e}$ is the electric vector potential of the dipole electric fi eld, which can be thought of as a dual to the normal magnetic vector potential of a magnetic dipole made of monopole-anti-monopole. By symmetry argument we can require the vector potential as $\vec{A}_{e}=A_{e}^{\phi}(\rho, z) \hat{\phi}$ and the monopole wave function as $\Psi=\psi(\rho, z) e^{i f \phi}$ with $f$ the z-angular-momentum quantum number. Then the stationary Schroedinger equation is simplifi ed to be

$$
\begin{aligned}
& {\left[\frac{\vec{p}_{\rho}^{2}+\vec{p}_{z}^{2}}{2 m}+V_{e f f}\right] \psi=E \psi} \\
& V_{e f f}=\frac{\hbar^{2}}{2 m}\left[\frac{1}{\rho / a}\left(\frac{g e}{\hbar} \frac{\rho A_{e}^{\phi}}{e}+f\right)\right]^{2}
\end{aligned}
$$

To go further one has to specify a gauge (which is equivalent to choosing some particular dual Dirac strings for the charges) so as to explicitly write down $A_{e}^{\phi}$. We use the gauge which corresponds to the situation with one Dirac string going from the positive charge along positive $\hat{z}$ axis to $+\infty$ and 


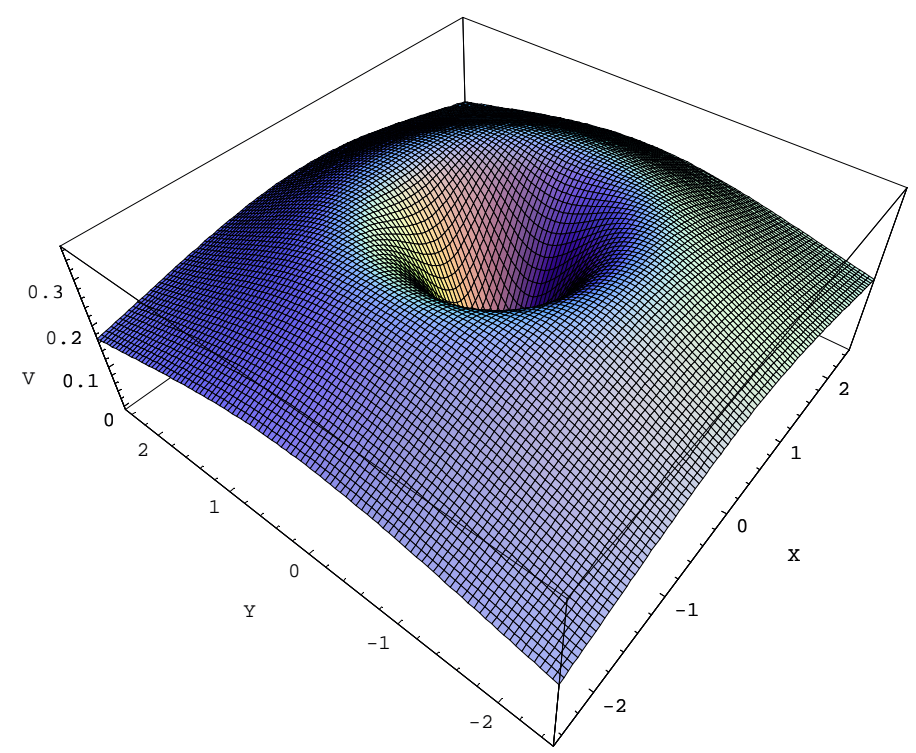

Figure 3: (color online) Quantum mechanical effective potential for a monopole in a static electric dipole field. See text for details.

the other going from the negative charge along negative $\hat{z}$ axis to $-\infty$. This gives us:

$$
A_{e}^{\phi}=-\frac{e}{\rho}\left[2+\frac{z-a}{\sqrt{\rho^{2}+(z-a)^{2}}}-\frac{z+a}{\sqrt{\rho^{2}+(z+a)^{2}}}\right]
$$

To give an idea of the effective potential we show Fig.3 where $V_{\text {eff }}(\rho, z, f)=V_{\text {eff }}\left(\sqrt{x^{2}+y^{2}}, z, f\right)$ is plotted for the $\mathrm{x}-\mathrm{y}$ plane with $z=0$ and $f=0$. From the plot we can see that there must also be quantum states with the monopole bounded within the potential well around the dipole for a long time before eventually decaying away.

\section{Molecular dynamics of electric/magnetic plasma}

If electric and magnetic quasiparticles can be described classically ${ }^{8}$, we know how do describe their ensemble, even at strong coupling. One should not use Boltzmann eqn or other simplistic transport, but use instead Molecular Dynamics (MD), which means solving numerically equations of motion. It is quite practical to do so to $\sim 1000$ particles and to abandon periodic boundary conditions: see the details in [10].

The summary of the sQGP transport properties is shown as a log-log plot of properly normalized dimensionless (heavy quark) diffusion constant and viscosity in Fig.1. The weakly coupled (gas) regime is in the left lower corner ${ }^{9}$. RHIC phenomenology (corresponding to the shaded oval) is however quite far from the weakly coupled regime. The AdS/CFT line ${ }^{10}$ on the other hand, neatly crosses through it, leading to optimistic conclusions. However our MD results, especially

\footnotetext{
${ }^{8}$ Which is possible as soon as their masses are large compared to $T$, which is approximately the case close to $T_{c}$. It is better satisfi ed for electric than magnetic ones, however.

${ }^{9}$ It has slope one since in this case both quantities are proportional to the same mean free path.

${ }^{10}$ Viscosity according to [15] with $O\left(\lambda^{-3 / 2}\right)$ correction, diffusion constant from [16].
} 


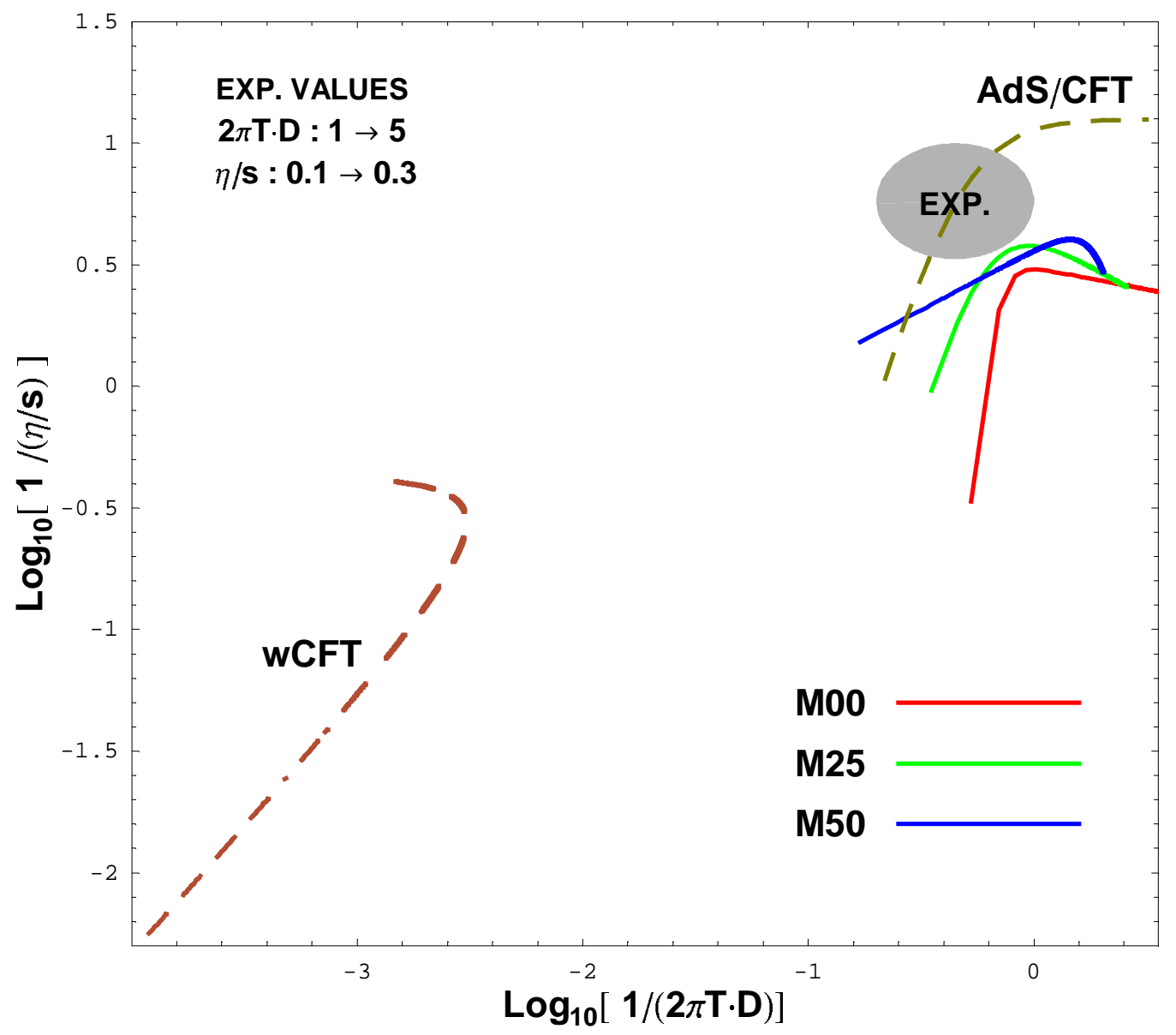

Figure 4: Plots of $\log [1 /(\eta / s)]$ v.s. $\log [1 /(2 \pi T D)]$ including results from our MD simulations, the Ads/CFT calculations, the weakly coupled CFT calculations, as compared with experimental values. M00,M25,M50 mean 0,25 and 50\% of monopoles in plasma.

for 50-50\% electric-magnetic plasma, are also in the right ballpark. We will discuss it a bit more in conclusions.

\section{Are there baryons above $T_{c}$ ?}

A talk at GSI cannot be without a special discussion of the FAIR physics domain, a dense baryonic matter. One issue I would like to discuss briefly at the end is put in the title of this section. Indeed, confi nement is not necessary for binding, and if some mesons survive the phase transition into the plasma phase, why not baryons?

The first thing to do is of course simply to use quantum mechanics and see in which domain the potentials between the QGP quasiparticles used for mesons would bind baryons as well. This was done some time ago in [17], with results summarized in Fig.5. One can see that while e.g. diquarks have negligible binding already at $T_{c}$, the s-wave baryons $-\mathrm{N}, \Delta \ldots-$ seem to survive up to $1.6 T c$. 


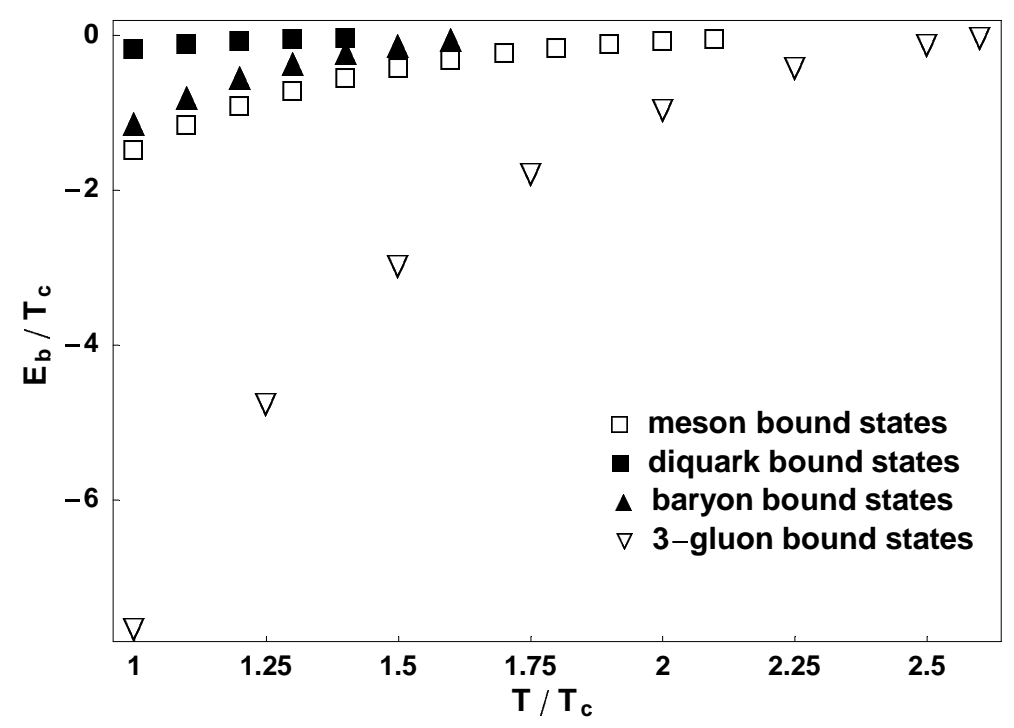

Figure 5: Dependence of various states' binding energy on the temperature. The units are in $T_{c}$.

The next step came from Bielefeld-UK collaboration [18] which calculated susceptibilities (up to sixth derivative over baryonic chemical potential $\mu_{B}$ and/or isospin one $\mu_{I}$ ) and thus provided intriguing information on the QGP thermodynamics at nonzero $\mu$. Unexpected "peaks" in the 4th and 'wiggles' in the 6-th were found in their $T$-dependence. Our analysis of these data [19] concluded that they can be naturally explained in a scenarios with surviving baryons, provided baryonic masses grow above $T_{c}$. Ratti,Roessner and Weise [20] have provided another impressive description of the same data in the PNJL model. In fact their conclusions are not that different from ours: indeed, the main role of their nonzero Polyakov loop is to suppress quarks and diquarks in favor of zero-triality $q q q$ states. Although the latter are not treated as such, they are effectively the baryons we used in our work [19].

In conclusion: we have reasons to believe that SQGP at FAIR will be mostly made of baryons, with a relatively small admixture of "ionized" quarks. Direct lattice investigations of baryonic correlation functions at fi nite $\mu$ will tell us more about their properties.

Fig.5 shows that the most robust baryon-like objects we found from binding calculations is made of three gluons. In fact $N_{c}(=3)$ adjoint objects bind a bit differently from quarks: instead of a junction to which all colors are attracted, there is a closed chain involving all colors cyclically closed to itself, e.g. $(\overline{\text { red }}-$ blue $)(\overline{\text { blue }}-$ green $)(\overline{\text { green }}-$ red $)$.

This talk, emphasizing importance of the electric-magnetic duality, should naturally end with a question of whether adjoint magnetic quasiparticles - monopoles and dyons - can or cannot form similar bound states? Not only so, but in fact these "dyonic baryons" (in which $N_{c}$ dyons are attracted to each other pair-vise, both electrically and magnetically) are already well known: in fact they are the fi nite- $T$ instantons - calorons - see [21]. On the lattice they have been seen and studied in multiple works ${ }^{11}$. The same construction can be obtained via a very interesting AdS/CFT picture with wrapping branes [22]. There is no doubt we will have a zoo full of exotic animals in the FAIR

\footnotetext{
${ }^{11}$ In fact chains up to 6 dyons with alternating colors have been seen, (M.Ilgenfritz, private communications).
} 
domain: the question is whether we will be smart enough to trace them down.

\section{Conclusions}

Theory of strongly coupled QGP has developed into two different brunches: one, based on AdS/CFT emphasizes quasi-conformal behavior and may be applicable at $T>1.5 T c$ or so; another emphasizes proximity to deconfi nement and changes in electric-magnetic equilibrium. As our brief "transport summary" in Fig.4 indicate that at the moment both may claim to be an explanation of RHIC data.

Thus we face a dilemma: Are these two explanations (i) deeply related and basically tell us the same story in two different languages, or (ii) there is no deep relation between them and we just see a mere coincidence of some transport coeffi cients? At the moment our understanding of AdS/CFT plasma at the microscopic level is too poor to tell.

The $\mathscr{N}=4$ SYM theory involved in AdS/CFT correspondence has, by itself, an electric-magnetic duality, in a form even much more perfect than QCD. It has Higgsed moduli spaces and magnetically charged supermultiplet, which happen to be the same as the original gauge-fermion-scalar one of the electric sector. However, in order to see electric-magnetic transitions we speak about one has to give up the large number of colors $N_{c} \rightarrow \infty$ limit, which is at this moment always used in practical AdS/CFT applications ${ }^{12}$.

Perhaps we will see resolution of this dilemma fi rst from experiment. If the LHC collisions turn out to be similar to RHIC ones, with hydro-predicted elliptic flow, it would mean we have sQGP in a quasi-conformal domain. If not and sQGP is only there near $T_{c}$, the electric-magnetic struggle would be the one left.

\section{References}

[1] E.V.Shuryak, "Why does the quark gluon plasma at RHIC behave as a nearly ideal fluid?," Prog. Part. Nucl. Phys. 53, 273 (2004) [ hep-ph/0312227].

[2] E.V.Shuryak and I. Zahed, hep-ph/0307267, Phys. Rev. C 70, 021901 (2004)

[3] E.V.Shuryak and I. Zahed, Phys. Rev. D69 (2004) 014011. [ hep-th/0308073].

[4] M. Gyulassy and L. McLerran, "New forms of QCD matter discovered at RHIC," Nucl. Phys. A 750, 30 (2005) [ nucl-th/0405013]. E. V. Shuryak,Prog.Part.Nucl.Phys.53:273-303,2004, hep-ph/0312227 "What RHIC experiments and theory tell us about properties of quark-gluon plasma?," Nucl. Phys. A 750, 64 (2005).

[5] G. D. Moore and D. Teaney, "How much do heavy quarks thermalize in a heavy ion collision?," hep-ph/0412346.

[6] H. Stoecker, "Collective Flow signals the Quark Gluon Plasma," Nucl. Phys. A 750, 121 (2005) [arXiv:nucl-th/0406018].

[7] J. Casalderrey-Solana, E. V. Shuryak and D. Teaney, "Conical fbw induced by quenched QCD jets," hep-ph/0411315. "Hydrodynamic fbw from fast particles," arXiv:hep-ph/0602183.

\footnotetext{
${ }^{12}$ It obscures the issue, making both electric and magnetic interactions infi nitely strong.
} 
[8] J. J. Friess, S. S. Gubser, G. Michalogiorgakis and S. S. Pufu, "The stress tensor of a quark moving through N = 4 thermal plasma,” Phys. Rev. D 75, 106003 (2007) [arXiv:hep-th/0607022].

S. S. Gubser, S. S. Pufu and A. Yarom, "Sonic booms and diffusion wakes generated by a heavy quark in thermal AdS/CFT," arXiv:0706.4307 [hep-th].

[9] P. M. Chesler and L. G. Yaffe, "The wake of a quark moving through a strongly-coupled $\mathscr{N}=4$ supersymmetric Yang-Mills plasma,” arXiv:0706.0368 [hep-th].

[10] J. Liao and E. Shuryak, "Strongly coupled plasma with electric and magnetic charges," Phys. Rev. C 75, 054907 (2007) [arXiv:hep-ph/0611131].

[11] S. Mandelstam, "Vortices And Quark Confinement In Nonabelian Gauge Theories," Phys. Rept. 23, 245 (1976).

G. 't Hooft, “Topology Of The Gauge Condition And New Confinement Phases In Nonabelian Gauge Theories," Nucl. Phys. B 190, 455 (1981).

[12] A. Nakamura, T. Saito and S. Sakai, "Lattice calculation of gluon screening masses," Phys. Rev. D 69, 014506 (2004) [hep-lat/0311024].

[13] O. Kaczmarek, S. Ejiri, F. Karsch, E. Laermann and F. Zantow, hep-lat/0312015.

[14] J. Liao and E. Shuryak, "Electric Flux Tube in Magnetic Plasma," arXiv:0706.4465 [hep-ph].

[15] G. Policastro, D. T. Son and A. O. Starinets, "The shear viscosity of strongly coupled N = 4 supersymmetric Yang-Mills plasma,” Phys. Rev. Lett. 87 (2001) 081601.

[16] J. Casalderrey-Solana and D. Teaney, "Heavy quark diffusion in strongly coupled N = 4 Yang Mills," hep-ph/0605199.

[17] J. Liao and E. V. Shuryak, "Polymer chains and baryons in a strongly coupled quark-gluon plasma," Nucl. Phys. A 775, 224 (2006) [arXiv:hep-ph/0508035].

[18] C. R. Allton et al., "Thermodynamics of two fhvor QCD to sixth order in quark chemical potential," Phys. Rev. D 71, 054508 (2005) [arXiv:hep-lat/0501030].

[19] J. Liao and E. V. Shuryak, "What do lattice baryonic susceptibilities tell us about quarks, diquarks and baryons at T > T(c)?,’ Phys. Rev. D 73, 014509 (2006) [arXiv:hep-ph/0510110].

[20] C. Ratti, S. Roessner and W. Weise, "Quark number susceptibilities: Lattice QCD versus PNJL model," arXiv:hep-ph/0701091.

[21] T. C. Kraan and P. van Baal, "Exact T-duality between calorons and Taub - NUT spaces," Phys. Lett. B 428, 268 (1998) [arXiv:hep-th/9802049].

[22] K. M. Lee and P. Yi, “Monopoles and instantons on partially compactified D-branes," Phys. Rev. D 56, 3711 (1997) [arXiv:hep-th/9702107]. 